\title{
Distribution longitudinale des macroinvertébrés benthiques de la Moulouya et de ses principaux affluents (Maroc)
}
A.Berrahou 1
B. Cellot ${ }^{2}$
P. Richoux ${ }^{2}$

Mots-clés : macroinvertébrés, Moulouya, Maroc, continuum fluvial, groupes trophiques.

L'étude a été réalisée début mai et fin juin 1994 dans 19 stations : 12 au long du cours principal et 7 au niveau de sources ou d'importants affluents. Les richesses taxonomique et spécifique (principalement Mollusques, Ephéméroptères, Plécoptères, Coléoptères et Trichoptères) montrent une évolution semblable avec un maximum à l'aval. L'analyse biotypologique par A.F.C. sur 68 espèces distingue 4 zones : le haut cours incluant les sources et les affluents montagnards, une zone de transition, une section moyenne très homogène avec les bas cours des principaux affluents malgré des perturbations polluantes et un bas cours à l'aval de deux barrages-retenues. Trois assemblages faunistiques majeurs se dégagent : un d'espèces du crénon-rhithron, un d'espèces plutôt eurytopes et thermophiles, et un d'espèces potamo-limnophiles. La zone de transition se définit alors par les deux assemblages entre lesquels elle se situe. Sur cette base typologique, la prise en compte des groupes trophiques montre que, seule, la basse Moulouya ne s'inscrit pas dans le schéma fonctionnel du 'R.C.C.'. La discontinuité observée à l'aval est probāblemènentdue aux effets des barrages (réduction du débit et de la largeur du lit), mais aussi au fait que cette section du fleuve retrouve un caractère plus naturel (ripisylve moins dégradée).

\section{Longidutinal distribution of benthic macroinvertebrates in the Moulouya River (Morocco)}

Keywords : macroinvertebrates, Moulouya, Morocco, river continuum, functional feeding groups.

The longitudinal distribution of benthic macroinvertebrates has been studied along the Moulouya River, one of the three permanent large rivers of Morocco. The study was carried out in May and June 1994 on 19 sites : 12 along the main channel and 7 on springs or important tributaries. Taxonomic richness and species richness (mainly Mollusca, Ephemeroptera, Plecoptera, Coleoptera and Trichoptera) showed similar patterns with a maximum downstream. Biotypologic analysis by C.A. performed on 68 species distinguished four sections : i) the upper Moulouya, including springs and mountain tributaries with crénobialrithral species, ii) a transitionnal zone, iii) the very homogeneous section of the middle Moulouya plus downstream zones of tributaries despite water pollution and characterized by eurytopic-thermophilous species, and finally iv) the lower Moulouya characterized by potamic-limnophilous species downstream from two successive impoundements. On this basis, the study of functional feeding groups showed that only the lower Moulouya did not correspond to the predictions of the River Continuum Concept. The discontinuity in the lowermost section was due to both regulation effects (discharge and streambed width reductions) and to a more abundant riparian vegetation.

1. Université Mohamed Premier, Faculté des Sciences, Département de Biologie, Laboratoire d'Hydrobiologie et Ecologie Générale, 60000 Oujda, Marqc.

2. Université Claude Bernard-Lyon 1, UMR CNRS 5023 Ecologie des Hydrosystèmes Fluviaux, 43 boulevard du 11 Novembre 1918,69622 Villeurbanne cedex, France. 


\section{Introduction}

Les écosystèmes d'eaux courantes présentent une grande diversité physique non seulement entre régions géographiques mais aussi de l'amont vers l'aval. Ainsi, plusieurs essais de zonation ou de typologie longitudinale, considérée surtout du point de vue faunistique, ont été proposés, notamment par Illies et Botosaneanu (1963), Verneaux (1973), et Botosaneanu (1979). Ces auteurs montrent que les différents paramètres mésologiques (pente, largeur du lit, substrat, débit, température, etc.) et la composition spécifiques des peuplements d'invertébrés benthiques révèlent des gradients voire des discontinuités écologiques au long des cours d'eau. Ces derniers peuvent être ainsi divisés en une série de zones se succédant depuis la source jusqu'à l'embouchure.

Vannote et al. (1980) font intervenir la notion de "groupe fonctionnel alimentaire" définie par Cummins (1973 et 1977) en mettant en relation le rôle des sources et modes de nutrition des macroinvertébrés avec la dynamique de la matière organique (Cummins \& Klug .1979)... Is formulent le principe du continuum fluviaal ou ."River Continuum Concept". (R.C.C.) qui considère-l'ajustement-des communautés biologiques des systèmes lotiques naturels dans un gradient continu de conditions physiques. Fortement discuté principalement sur ce dernier point, notamment par Statzner et Higler (1985) (voir aussi la revue critique de Wasson, 1989), le concept a été élargi de façon à prendre en compte des facteurs importants tels que le climat, la géologie, les affluents, la lithologie, la géomorphologie, et aussi les perturbations humaines qui peuvent modifier le schéma du 'RCC' (Minshall et al. 1985). Même sous l'influence de ces facteurs, l'évolution longitudinale de la richesse des macroinvertébrés reste prédite pour être maximale dans le tronçon moyen (autour de l'ordre 4). Les auteurs réaffirment également la substitution des dominances des broyeurs-déchiqueteurs, puis des racleurs-brouteurs et enfin des collecteurs-filtreurs d'amont en aval. C'est cette idée qui a été testée principalement en Amérique (voir Minshall et al. 1985), en Australie (Marchant et al. 1985, Bunn 1986) et en Europe (Rosillon 1986). Mais ces études, souvent restreintes à de petites rivières, n'ont confirmé que partiellement le concept. Des facteurs régionaux et locaux sembleraient plus importants pour prévoir et expliquer le fonctionnement biologique d'un tronçon que la position de ce dernier dans le réseau fluvial (Wasson 1989).

L'objectif du présent travail est de tester les prédictions du 'R.C.C.' concernant la diversité et la structure fonctionnelle de la communauté des invertébrés benthiques, pour la première fois à notre connaissance tout au long d'un grand fleuve d'Afrique du nord. Cette étude réalisée sur le bassin de la Moulouya, s'inscrit dans l'effort de recherche, nécessaire pour la limnologie marocaine (Chergui et al. 1999) et plus généralement, dans le cadre des eaux courantes des régions à climat méditerranéen (Gasith \& Resh 1999).

\section{Site d'étude, matériel et méthode}

\subsection{La Moulouya}

La Moulouya est le seul grand fleuve marocain se jetant dans la Méditerranée (Fig. 1). Long de plus de $500 \mathrm{~km}$, il draine la quasi-totalité du Maroc oriental (bassin versant d'environ $53000 \mathrm{~km}^{2}$ ). Ce cours d'eau prend sa source à $1855 \mathrm{~m}$ d'altitude dans la zone de jonction du Haut Atlas - Moyen Atlas et reçoit assez rapidement deux affluents montagnards (l'Oudghes et l'Anzegmir) puis successivement, le Melloulou et le $\mathrm{Za}$; deux barrages-retenues (Mohamed V et Mechra-Homadi) sont implantées dans des gorges profondes avant son embouchure (Reynal 1961). Le fleuve traverse divers étages bioclimatiques méditerranéens qui sont fonction de l'altitude et de la continentalité (Sauvage 1963, El Gharbaoui 1987, El Gasmi 1987, Chergui et al. 1999).

La Moulouya a un régime hydrologique pluvial de type méditerranéen, caractérisé par des hautes eaux en fin d'hiver - début du printemps et par des basses eaux en été (Fig. 1). Le débit moyen annuel est de $3,3 \mathrm{~m}^{3} \cdot \mathrm{s}^{-1}$ à Zaïda, de 7,4 à Missour, de 20,9 à Guercif et de 23,0 juste après la confluence du Za. Les principaux affluents que sont l'Anzegmir, le Melloulou et le Za, présentent en effet des modules annuels non négligeables (respectivement : 2,$9 ; 9,5$ et $3,6 \mathrm{~m}^{3} \cdot \mathrm{s}^{-1}$ ). Leurs régimes hydrologiques très irréguliers, avec un étiage estival sévère et des crues violentes aux autres saisons, jouent fortement sur les fluctuations de débit de la Moulouya. A l'aval des deux barrages, du fait de l'évaporation et de très importants prélèvements d'eau dans les retenues pour l'irrigation, le débit moyen de la Moulouya est réduit de moitié $\left(12,0 \mathrm{~m}^{3} \cdot \mathrm{s}^{-1}\right)$, soit un débit proche de celui enregistré à l'amont de la confluence du Melloulou. De même, les fluctuations de débit sont très faibles au regard de celles enregistrées à l'amont des barrages. Ainsi, ce faible débit régularisé ne correspond plus aux caractéristiques naturelles de l'aval de ce fleuve.

Les principales caractéristiques physico-chimiques sont présentées dans le Tableau 1 . Il montre que les eaux de la Moulouya sont fortement minéralisées et 


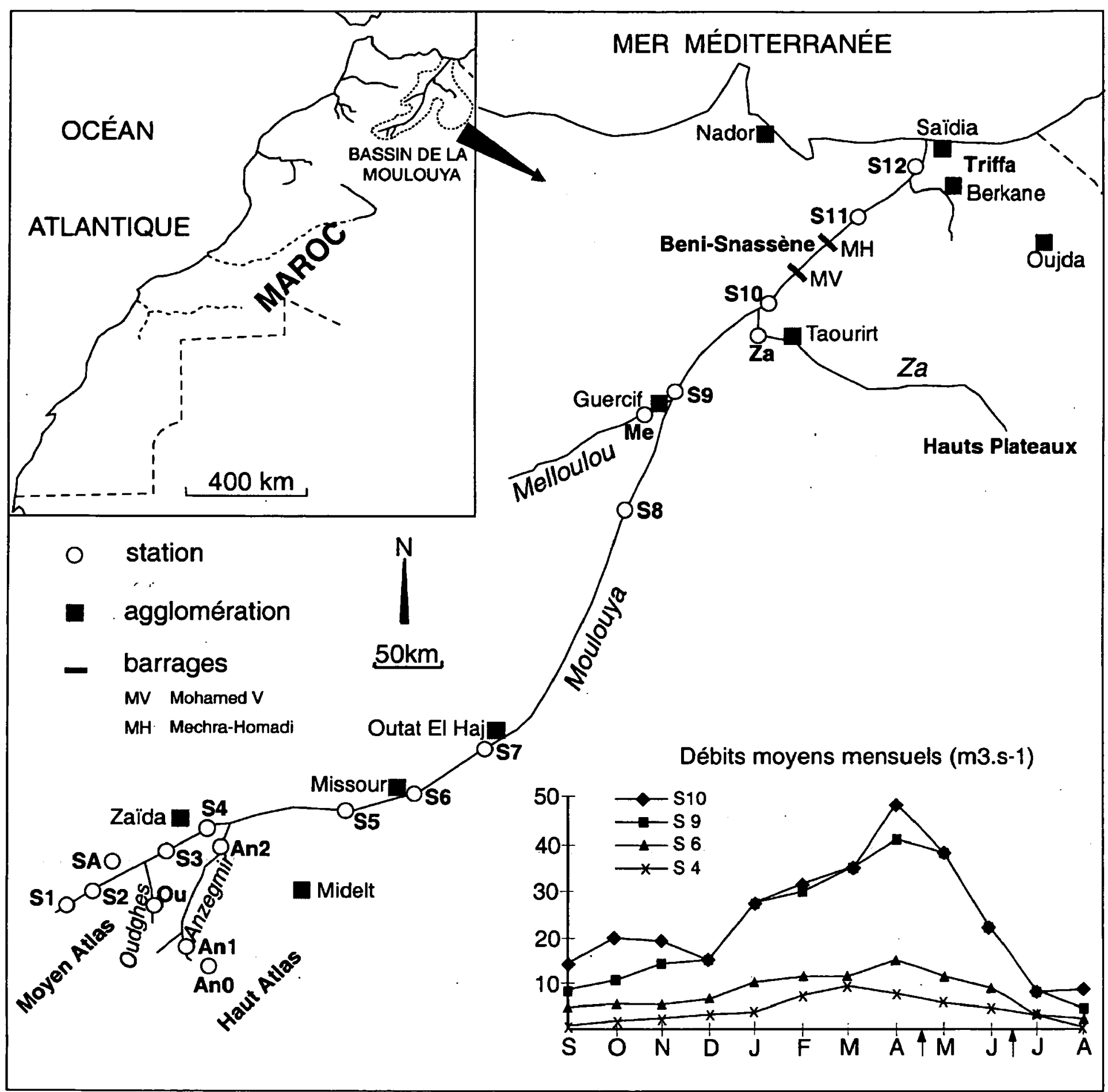

Fig. 1. Carte du bassin de la Moulouya avec la localisation des stations d'étude et l'évolution des débits moyens mensuels sur la période de référence de 1959-1989 (données de la Direction Régionale de l'Hydraulique d'Oujda).

Fig. 1. Map of the Moulouya watershed, location of the study sites and changes in monthly means of the discharges over the period 1959-1989 (data from Oujda's D.R.H.).

que les perturbations par les eaux usées des principales agglomérations (Zaïda en S4, Missour en S6, Guercif en S9 et Taourirt sur le Za) sont importantes.

\subsection{Le macrobenthos}

19 stations de prélèvements du macrobenthos (dont 10 correspondent à celles des données physico-chimiques) ont été retenues dans le bassin de la Moulouya (Fig. 1). Elles ont été choisies, soit à l'aval des princi- paux affluents du fleuve, soit à proximité des principales agglomérations afin de prendre en compte l'influence de ces affluents et l'impact probable des activités humaines. Douze stations sont situées le long du fleuve (S1-S12) et 7 au niveau de sources importantes (SA, An0) ou des principaux affluents (Ou, An1, An2, $\mathrm{Me}, \mathrm{Za})$. Ces stations présentent des faciès assez contrastés (Tab. 2). Ainsi, celles de haute altitude $(>1400 \mathrm{~m}$ ) possèdent une végétation aquatique et une 
Tableau 1. Physico-chimie de la Moulouya et de ses principaux affluents ; valeurs ponctuelles mesurées du 20 avril au 21 mai 1992 par la 'Direction Régionale de l'Hydraulique' pendant une période de stabilité hydraulique (données D.R.H. d'Oujda, Maroc).

Table 1. Physical and chemical features of the Moulouya River and its main tributaries ; single values collected during stable flows from 20 April to 21 May 1992 by the 'Direction Régionale de l'Hydraulique' (data from Oujda's D.R.H., Morocco).

\begin{tabular}{|c|c|c|c|c|c|c|c|c|c|c|c|c|}
\hline Station & Code & $\begin{array}{c}\text { Temp. } \\
{ }^{\circ} \mathrm{C}\end{array}$ & $\mathrm{pH}$ & $\begin{array}{l}\text { Cond. } \\
20^{\circ} \mathrm{C}\end{array}$ & $\begin{array}{c}\mathrm{O} 2 \\
\mathrm{mg} / 1\end{array}$ & $\begin{array}{c}\text { O2 } \\
\text { \% Sat. }\end{array}$ & $\begin{array}{c}\mathrm{DCO} \\
\mathrm{mg} \mathrm{O} 2 / 1\end{array}$ & $\begin{array}{l}\mathrm{NO} 3 \\
\mathrm{mg} / \mathrm{l}\end{array}$ & $\begin{array}{l}\mathrm{NO} 2 \\
\mathrm{mg} / \mathrm{l}\end{array}$ & $\begin{array}{l}\mathrm{NH} 4 \\
\mathrm{mg} / \mathrm{l}\end{array}$ & $\begin{array}{c}\mathrm{Cl} \\
\mathrm{mg} / \mathrm{l}\end{array}$ & $\begin{array}{c}\mathrm{Na} \\
\mathrm{mg} / \mathrm{l}\end{array}$ \\
\hline \multicolumn{13}{|l|}{$\overline{\text { MOULOUYA }}$} \\
\hline Zaïda & S4 & 24 & 7.6 & 620 & 7.2 & 85 & 16 & 11.26 & 0.24 & 0.03 & 35.45 & 18.5 \\
\hline Aval Missour & S6 & 25 & 7.9 & 910 & 6.2 & 75 & 32 & 6.20 & 0.26 & 0.07 & 31.90 & 25.0 \\
\hline Outat El Haj & S7 & 25 & 7.6 & 1005 & 7.0 & 85 & 12 & 9.92 & 0.05 & 0.07 & 88.60 & 55.0 \\
\hline Tindit & S8 & 25 & 7.7 & 1215 & 6.4 & 78 & 8 & 7.76 & 0.06 & 0.08 & 88.63 & 40.0 \\
\hline Guercif & s9 & 23 & 7.0 & 995 & 5.0 & 58 & 14 & - & 0.29 & 0.08 & 81.00 & 70.0 \\
\hline Melga El Ouidane & S10 & 23 & 7.8 & 1850 & 8.4 & 99 & 8 & 9.84 & 0.47 & 0.09 & 276.50 & 140.0 \\
\hline Safsaf & S11 & 20 & 7.2 & 2100 & 8.0 & 89 & 12 & 11.40 & 0.03 & 0.01 & 382.00 & 215.0 \\
\hline \multicolumn{13}{|l|}{ AFFLUENTS } \\
\hline Anzegmir & An2 & 24 & 7.2 & 415 & 7.4 & 88 & 4 & 7.68 & 0.05 & 0.03 & 21.30 & 11.5 \\
\hline Melloulou & Me & 23 & 7.2 & 670 & 7.2 & 84 & 10 & 5.90 & 0.03 & 0.05 & 56.70 & 50.0 \\
\hline $\mathrm{Za}$ & $\mathrm{Za}$ & 23 & 7.6 & 1935 & 8.2 & 95 & 25 & 10.17 & 0.50 & 0.15 & 301.00 & 130.0 \\
\hline
\end{tabular}

Tableau 2. Localisation, code et caractéristiques des stations d'étude faunistique de la Moulouya et de ses principaux affluents.

Table 2. Location, code, and characteristics of the benthic macroinvertebrate sampling sites in the Moulouya River and its main tributaries.

\begin{tabular}{|c|c|c|c|c|c|}
\hline Station & Code & $\begin{array}{l}\mathrm{km} \text { à la } \\
\text { source }\end{array}$ & $\begin{array}{l}\text { Altitude } \\
(\mathrm{m})\end{array}$ & $\begin{array}{l}\text { Largeur } \\
\text { (m) }\end{array}$ & $\begin{array}{l}\text { Substrat des fonds et } \\
\text { végétation des rives }\end{array}$ \\
\hline \multicolumn{6}{|l|}{ MOULOUYA } \\
\hline Tifratine & S1 & 20 & 1655 & 2 & Bloc, galet, gravier et Juncus, Typha \\
\hline Bou Irsane & S2 & 27 & 1615 & 2 & Bloc, gravier et Juncus, Typha, Salix, Populus \\
\hline Boumia & S3 & 53 & 1520 & 3 & Galet, gravier et Juncus, Typha, Salix \\
\hline Zaïda & S4 & 68 & 1470 & 4 & Dalle, bloc, galet et Typha, herbes \\
\hline Tamdafelt & S5 & 130 & 1000 & 26 & Pierre plate, galet et Tamarix \\
\hline Missour & S6 & 164 & 875 & 25 & Galet, gravier, sable et rares Tamarix, Populus \\
\hline Outat El Haj & S7 & 206 & 700 & 25 & Galet, gravier, sable et rares Tamarix \\
\hline Tindit & S8 & 346 & 625 & 90 & Bloc, galet, sable \\
\hline Guercif & s9 & 405 & 280 & 75 & Galet, gravier et rares Tamarix \\
\hline Melga El Ouidane & S10 & 443 & 240 & 100 & Bloc, galet, gravier, sable \\
\hline Safsaf & S11 & 496 & 50 & 39 & Galet et Potamogeton, Chara, Typha, Juncus, Tamarix, Nerium \\
\hline Cap de l'Eau & S12 & 527 & 20 & 44 & Galet, gravier, sable et Chara, Juncus, Tamarix \\
\hline \multicolumn{6}{|l|}{ SOURCES et AFFLUENTS } \\
\hline Aghbalou N'Serdane (source) & SA & & 1645 & 4 & Galet, aménagement pour eau potable \\
\hline Oudghes & Ou & & 1825 & 2 & Galet, gravier et Salix, rares Populus \\
\hline Anzar Oufounes (source) & Ano & & 1855 & 3 & Galet et Juncus, Chara \\
\hline Touha & Anl & & 1785 & 3 & Bloc, galet, gravier et Salix, rares Populus \\
\hline Anzegmir & An2 & & 1470 & 6 & Galet, gravier et Typha, Juncus, Tamarix \\
\hline Melloulou & Me & & 285 & 15 & Bloc, galet, gravier, sable-limon et rares Nerium \\
\hline $\mathbf{Z a}$ & $\mathrm{Za}$ & & 242 & 8 & Galet, gravier et rares Typha \\
\hline
\end{tabular}


ripisylve abondantes; celles de plus basse altitude n'ont que peu de végétation, mais les 2 dernières stations de la Moulouya retrouvent une végétation aquatique et une végétation terrestre denses dans le lit de cette section par suite d'une largeur en eau fortement réduite sous l'influence des retenues juste à l'amont.

Les relevés quantitatifs du macrobenthos ont été réalisés du 2 au 4 mai 1994 et du 23 au 26 juin 1994. Ces campagnes de prélèvements correspondent respectivement à une période de hautes eaux et à la période d'étiage dans le bassin de la Moulouya (Fig. 1). Elles correspondent également à la période de forte productivité biologique au Maroc, permettant ainsi d'obtenir une bonne représentation faunistique amont-aval (Dakki 1986b). La technique du filet de type Surber, avec un vide de maille de $0,4 \mathrm{~mm}$, a été utilisée. Six échantillons de $0,025 \mathrm{~m}^{2}$ dans des faciès différents (3 lotiques et 3 lénitiques) ont été réalisés par station et à chaque campagne, soit $0,3 \mathrm{~m}^{2}$ de surface inventoriée par station. Les échantillons ont été formolés sur le terrain puis triés au laboratoire sous la loupe binoculaire. La faune a été identifiée à l'espèce (excepté les Oligochètes, les Odonates, les Hétéroptères et les Diptères) et dénombrée par station en cumulant les données des deux campagnes (Annexe).

Les richesses taxonomique et spécifique ainsi que l'abondance par station ont d'abord été considérées. La richesse taxonomique ne fait pas intervenir les individus juvéniles non identifiés (Gammaridae, Baetidae et Hydropsychidae) et la richesse spécifique ne prend en compte que les 68 espèces déterminées sur les 84 taxons (Annexe). L'abondance correspond en revanche à la totalité des individus récoltés et ramenés au $\mathrm{m}^{2}$. Pour exprimer la biotypologie stationnelle (Dakki 1985), nous avons utilisé l'Analyse Factorielle des Correspondances (AFC) ; cette méthode d'ordination largement utilisée, fournit des scores factoriels qui peuvent être cartographiés (Dolédec \& Chessel 1991). Seules, les 68 espèces dont le code figure en Annexe, soit la très grande majorité des taxons, ont été prises en cômpte. Afin de réduire les disparités numériques entre ces variables, les abondances ont été transformées en classes d'effectifs de progression géométrique de raison 2. Sur la base de cette typologie stationnelle au niveau spécifique, l'abondance relative des groupes trophiques pour l'ensemble du peuplement au long de la Moulouya a été comparée avec celle prédite par le 'R.C.C.'. Les 4 groupes trophiques utilisés par ce dernier ('collectors' $=$ collecteurs filtreurs et fouisseurs ; 'grazers' = brouteurs et racleurs, 'predators' $=$ prédateurs et parasites ; 'shredders' = broyeurs et déchiqueteurs) sont renseignés en Annexe, à partir des informa- tions de Tachet et al. (2000) et en tenant compte de proportions pour les taxons faiblement identifiés (Oligochètes, certains Diptères) ou à régimes variés selon les stades (certains Coléoptères par exemple).

\section{Résultats}

\subsection{Richesse et abondance}

La richesse et l'abondance des macroinvertébrés montrent des fluctuations au long de la Moulouya (Fig. 2). Les variations des richesses taxonomique et spécifique sont très similaires, si bien que la richesse taxonomique apparaît suffisante pour décrire l'évolution amont-aval de la diversité dans le bassin de la Moulouya.

La richesse taxonomique (Fig. 2), faible en S1 et S2 avec respectivement 12 et 15 taxons, double en S3 avec 26 taxons. Cette forte augmentation est peut-être liée aux apports de faune dus à la source latérale limnocrène $(\mathrm{SA})$ et au premier affluent montagnard, l'Oudghes $(\mathrm{Ou})$. La richesse diminue fortement après Zaïda (de 26 en S3 à 19 en S4) et après Missour (de 20 en S5 à 13 en S6) ; l'apport de l'Anzegmir n'infléchit pas la diversité faunistique, étant lui-même pauvre (18 taxons en An1, 9 en An2). Corrélativement à ces chutes de richesse en aval de ces deux agglomérations, on observe des pics d'abondance (5317 ind. $\mathrm{m}^{-2}$ en S4 et 6940 en S6) alors que les stations S1, An1 et S7 dépassent tout juste 3600 ind. $\mathrm{m}^{-2}$ (Fig. 2). Une nouvelle chute de la richesse apparaît en aval de Guercif (10 taxons en S9) malgré l'apport potentiel du Melloulou (13 en Me). On constate, de S6 à S8, une chute de l'abondance qui continue à l'aval de l'agglomération de Guercif, contrairement à ce qui a été vu précédemment. Après la confluence du $\mathrm{Za}$, la richesse et l'abondance croissent malgré les bouleversements anthropiques du bas cours pour atteindre les valeurs maximales de la richesse (29 taxons en S11 et 33 en S12).

\subsection{Biotypologie stationnelle}

La figure 3 présente les résultats de l'AFC réalisée sur la matrice des 19 stations x 68 espèces (voir Annexe).

L'essentiel de la typologie des stations s'exprime sur les trois premiers axes factoriels, F1, F2 et F3, ceux présentant le pourcentage d'inertie les plus élevés (Fig. 3a). L'axe F1 oppose nettement les stations du haut cours de la Moulouya (SA, An0, S1, S2, Ou, An1) à l'ensemble des autres stations, à l'exception de S3 et S4. L'axe F2 oppose les stations de la Basse-Moulouya (S11 et S12) notamment aux stations de la MoyenneMoulouya qui avec les stations de bas cours des af- 

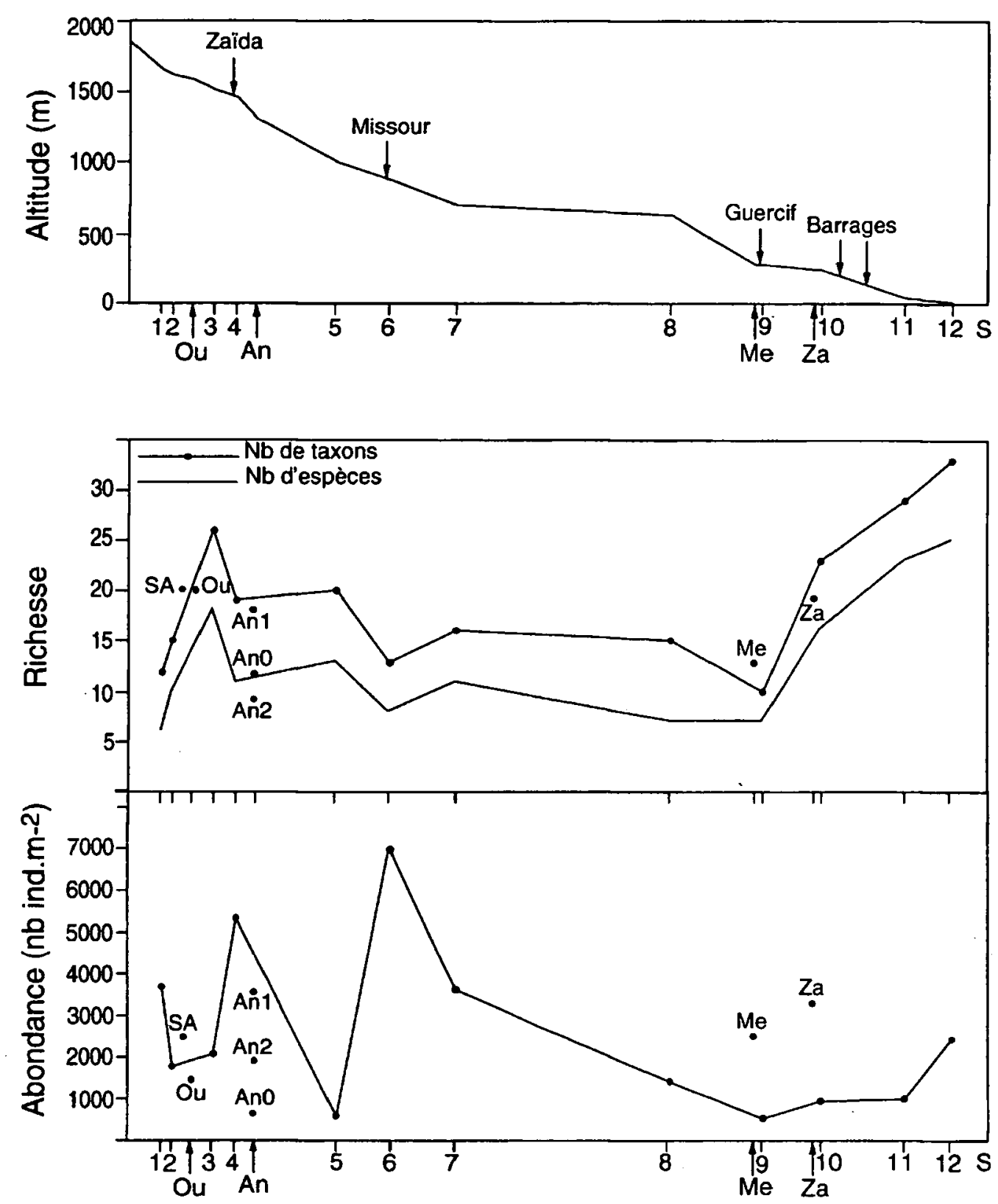

Fig. 2. Profil de la Moulouya d'après 12 stations (S), avec indications des confluences des principaux affluents (Ou, $\mathrm{An}, \mathrm{Me}, \mathrm{Za}$ ) et de perturbations importantes (villes, barrages) ; richesse et abondance totale des macroinvertébrés par station dans le bassin de la Moulouya.

Fig. 2. Altitudinal and longitudinal location of the 12 sites $(\mathrm{S})$ with confluences of main tributaries $(\mathrm{Ou}, \mathrm{An}, \mathrm{Me}, \mathrm{Za})$ and anthropic disturbances (cities, dams) ; richness and total abundance of macroinvertebrates collected in the Moulouya catchment.

fluents, sont très groupées dans le plan. L'axe F3, non représenté ici, ne fait apparaître que la distinction des stations S3 et S4 par rapport à l'ensemble des autres stations. Cela se retrouve d'ailleurs dans le plan F1xF2 où $\mathrm{S} 3$ et $\mathrm{S} 4$ se placent en position intermédiaire entre la Haute et la Moyenne-Moulouya. Face à l'homogénéité de la Moyenne-Moulouya, soulignons la séparation relative au long de F1, des sources (SA et An0) et du haut cours des affluents montagnards (Ou et An1). Cependant il est évident que sur $\mathrm{F} 1 \mathrm{xF} 2$, la variabilité des coordonnées des stations du haut cours de la Moulouya (variance intra-groupe) reste bien inférieure à celle que l'on observe entre les positions moyennes des groupes décrits (variance inter-groupe : Haute-Moulouya, secteur de transition, Moyenne-Moulouya et Basse-Moulouya).

Dans le plan F1xF2 des variables (Fig. 3b), si l'on fait exception des espèces qui discriminent par ailleurs les stations S3 et S4 (Lymnaea peregra (12), Choroterpes picteti (17), Laccophilus hyalinus (37) entreautres, (voir Annexe)), il est possible de repérer visuellement trois groupes faunistiques se distinguant d'amont en aval. Le groupe $\mathrm{A}$, avec 21 espèces dont les 

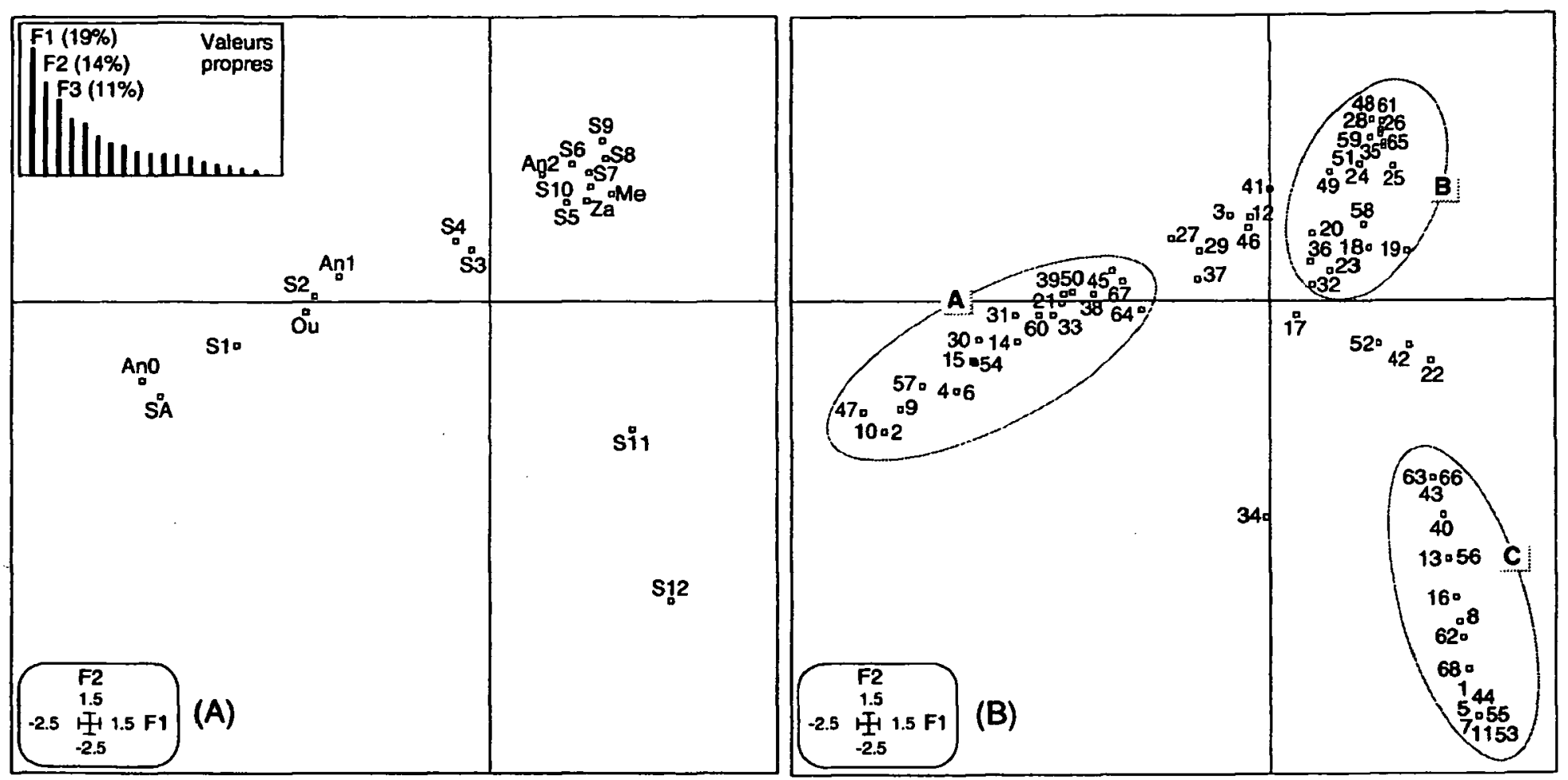

Fig. 3. Résultats du plan F1xF2 de l'analyse factorielle des correspondances réalisée sur la matrice 19 stations x 68 espèces (Annexe). (A) - plan pour les stations ; le pourcentage d'inertie sur les 3 premiers axes est donné par l'histogramme des valeurs propres. (B) plan pour les espèces (code figurant en Annexe).

Fig. 3. Results of the F1xF2 factorial map from a correspondence analysis performed on the matrix 19 sites and 68 species (Appendix). (A) - Map for the sites; percentage of inertia for the first 3 axes is given by the histogram of eigenvalues. (B) - Map for the species (see code in Appendix).

Crustacés Gammaridae, des Achètes, des Mollusques et des Insectes, correspond aux espèces de la HauteMoulouya ; certaines caractérisent par leur abondance, soit les sources comme Gammarus gauthieri (15), soit le haut rhitron comme Baetis rhodani (21) (voir Annexe); on note également la présence d'espèces à la fois crénophiles et rhitrophiles, telles que : Ancylus fluviatilis (9), Perla marginata (31), Oulimnius fuscipes (57) et Hydropsyche pellucidula (60). Le groupe B, avec 18 espèces, ne comprend par contre que des Ephéméroptères, des Coléoptères et des Trichoptères caractérisant la Moyenne-Moulouya; citons Caenis luctuosa (18), Baetis pavidus (20) et Hydropsyche maroccana (58) qui montrent ici une très large distribution longitudinale et, dans une moindre mesure, Oligoneuriopsis skounate (25), Ecdyonorus rothschildi (26), Herophydrus musicus (35), Hydropsyche lobata (59) et Hydropsyche resmineda (61) (voir Annexe). Le groupe $\mathrm{C}$ traduit un clivage avec les groupes précédents en ne caractérisant que la Basse Moulouya ; en effet sur les 17 espèces de ce groupe, 15 sont exclusives des deux dernières stations, dont les plus abondantes sont : Theodoxus fluviatilis (5), Melanopsis praemorsa (7), Melanopsis costellata (8), Atyaephyra desmarestii (16), Psychomyia pusilla (62) et Mystacides azurea (68) (voir Annexe).

La structure biotypologique des 19 stations montre donc l'existence de 4 zones distinctes : la Haute-Moulouya au sens strict (S1 et S2), à laquelle se rattachent d'une part les sources (SA+An0) et d'autre part le haut cours des affluents montagnards (Ou+An1) ; un secteur de transition ( $\mathrm{S} 3$ et $\mathrm{S} 4)$; le tronçon homogène de la Moyenne-Moulouya (S5 à S10, incluant le bas cours des principaux affluents $\mathrm{An} 2+\mathrm{Me}+\mathrm{Za}$ ) ; et enfin la Basse-Moulouya ( $\mathrm{S} 11$ et $\mathrm{S} 12$ ) très particulière en aval des retenues.

\subsection{Typologie et groupes trophiques}

Basée sur la typologie stationnelle, la figure 4 présente l'évolution des abondances relatives des groupes fonctionnels trophiques dans le bassin de la Moulouyá, suivant la forme, à titre comparatif, de celle du 'R.C.C.'. La comparaison distingue en correspondance d'un niveau donné, les stations de la Moulouya d'une part, de celles des sources et affluents d'autre part.

Ainsi, la première ligne de la figure 4 montre que l'importance relative des groupes trophiques change d'amont en aval. La Haute-Moulouya (S1+S2) est do- 

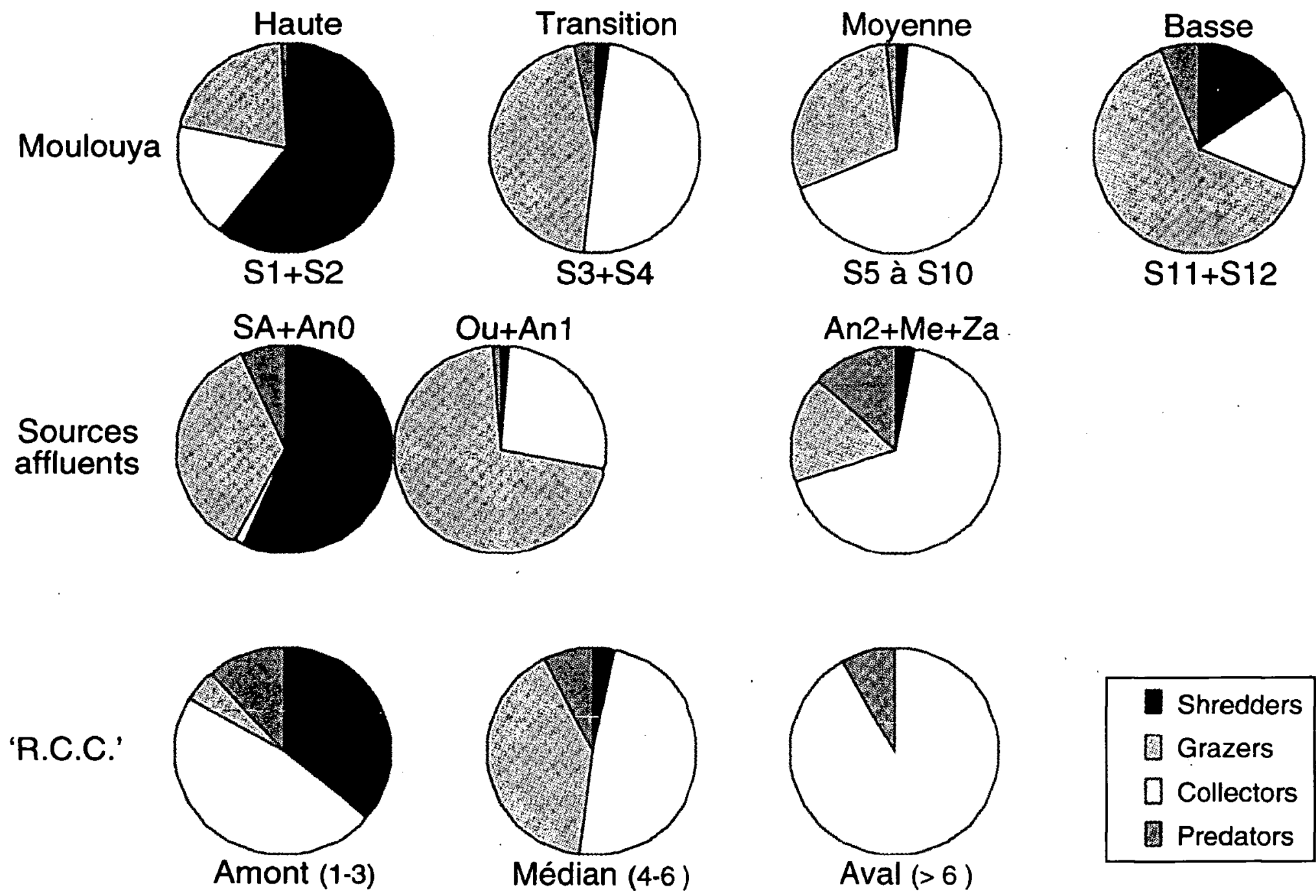

Fig. 4. Abondances relatives (en \%) des quatres groupes trophiques au long de la Moulouya, comparées aux prédictions du continuum fluvial ('R.C.C.').

Fig. 4. Relative abundances (\%) of the functional feeding groups along the Moulouya River, compared with predictions of the 'River Continuum Concept' (R.C.C.).

minée par les 'shredders' (60\%). L'essentiel de la communauté est constitué de 'collectors' et de 'grazers' en proportions à peu près égales (49 et $45 \%$ ) dans la zone de transition ( $\mathrm{S} 3+\mathrm{S} 4)$, mais ensuite, les 'collectors' (avec $67 \%$ ) dominent nettement sur les 'grazers' (29\%) dans la Moyenne-Moulouya (S5 à S10). Les 'grazers' (avec $63 \%$ ) sont à leur tour bien réprésentés dans la Basse-Moulouya (S11+S12) où la part des 'collectors' (15\%) et des 'shredders' (15\%) n'est pas négligeable. La deuxième ligne de la figure 4 met en évidence une certaine ressemblance entre la Haute-Moulouya et les sources (SA+An0), mais pas avec le haut cours des affluents d'altitudes (Ou+An1) du fait ici d'une surabondance nette de 'grazers' (71\%): On constate également la bonne similitude entre la Moyenne-Moulouya et les bas cours des principaux affluents (An2+Me+Za).

La comparaison avec les prédictions du R.C.C.; données en troisième ligne de la figure 4 , montre en défi- nitive une bonne correspondance entre l'amont théorique (ordres 1-3) et la Haute-Moulouya (sources comprises) ainsi qu'entre l'aval théorique (ordres $>6$ ) et la Moyenne-Moulouya (bas cours des affluents compris). La zone de transition, quant à elle, correspond parfaitement au secteur médian théorique (ordres 4-6). En revanche, le haut cours des affluents montagnards et la Basse-Moulouya ne présentent que peu de correspondances. S'il est possible de rapprocher les affluents d'altitude de la zone de transition, donc du secteur médian du 'R.C.C.', il apparaît toutefois assez difficile d'intégrer la Basse-Moulouya dans le continuum fluvial.

\section{Discussion}

Du point de vue biogéographique, la macrofaune du bassin de la Moulouya appartient à la zone paléarctique avec des espèces endémiques marocaines ou 
maghrébines, notamment : Ecdyonurus rothschildi, Hydropsyche maroccana, Hydropsyche resmineda, Nebrioporus clarki, Hydraena rigua, Normandia villosocostata. Cette communauté benthique est marquée par la rareté des Plécoptères. L'absence de ce groupe dans certains cours d'eau d'Afrique du Nord semble être due aux températures estivales élevées en relation avec le cycle hydrologique. Plusieurs travaux (Giudicelli \& Dakki 1984, Dakki 1986a\&b, Bouzidi 1989, El Agbani et al. 1992) mentionnent que les Plécoptères se limitent aux seules eaux courantes bien oxygénées de haute altitude. C'est un peu le cas dans la Moulouya, mais la rareté des Plécoptères pourrait être aussi due au manque de débris organiques (Chergui et al. 1990, Maamri et al. 1994), la pauvreté en végétation aquatique et riveraine caractérisant le cours moyen de la Moulouya. En effet, Lounaci et al. (2000) ont montré que l'abondance des Plécoptères au long d'une rivière algérienne était liée à l'importance de la ripisylve. Quoi qu'il en soit, la macrofaune étudiée dans la Moulouya est surtout caractérisée par une forte diversité spécifique des Coléoptères (Berrahou et al. 2000, 2001). Comparée aux autres cours d'eau du Maroc, la Moulouya ressemble plutôt au Bou Regreg qui présente un régime globalement temporaire, un fort réchauffement de ses eaux en période sèche, un faible débit d'étiage et un peuplement dominé par des espèces thermophiles (El Agbani et al. 1992, Chergui et al. 1999).

Du point de vue longitudinal, le modèle des richesses observées dans le bassin de la Moulouya ne correspond pas aux prédictions du 'R.C.C.' (Vannote et al. 1980, Minshall et al. 1985). L'évolution parallèle de la richesse taxonomique et spécifique (principalement celle des Mollusques, Ephéméroptères, Plécoptères, Coléoptères et Trichoptères) s'accorde plutôt avec les propositions de Statzner \& Higler (1986) sur la zonation des rivières originelles. En effet, après une richesse faible au niveau des sources et de la Haute-Moulouya qui s'explique par les conditions thermiques contrastées en altitude dans les cours d'eau du HautAtlas (Giudicelli \& Dakki 1984, Bouzidi 1989), la diversité tend très vite à augmenter après la confluence avec le premier affluent montagnard (Oudghes). Un tel schéma est décrit par Statzner \& Higler (1986) au niveau d'une première transition de la contrainte hydraulique où les espèces des sources et celles du secteur à fortes pentes se recouvriraient. Ensuite, la Moulouya est trop sous l'emprise de perturbations anthropiques et de pollutions locales (Chergui et al. 1999) pour mettre en évidence une deuxième transition hypothétique de même type au niveau du piémont. Cependant, après une diversité faible, Statzner \& Higler (1986) propo- sent de nouveau une richesse accrue voire maximale à l'aval, et c'est bien ce qui se passe sur la Moulouya. Après les barrages-retenues, le tronçon aval retrouve une végétation aquatique diversifiée dans un cours réduit qui s'inscrit dans un lit majeur colonisé par une abondante végétation riveraine. Associé à la régularisation des débits, cela entraîne une augmentation de l'hétérogénéité des habitats physiques, notamment près des rives qui constituent des lieux de ponte et de refuge pour les invertébrés aquatiques des grandes rivières (Cellot et al. 1984 ; Bournaud \& Cogérino 1989, Cogérino et al. 1995).

L'analyse biotypologique du bassin de la Moulouya montre des différences amont-aval rencontrées souvent dans les eaux courantes, en particulier méditerranéennes (Giudicelli et al. 1980, Gonzalez del Tanago \& Garcia de Jalon 1981, Dia 1983, Giudicelli et al. 1985, El Agbani et al. 1992). La Haute-Moulouya et ses sources sont caractérisées par de nombreuses espèces plutôt sténothermes, comme Gammarus gauthieri ou Beatis rhodani qui est considéré comme sténotherme froid au Maroc (Dakki 1986b). La Moyenne Moulouya et les bas cours des principaux affluents ne se distinguent que par des Ephéméroptères, des Coléoptères et des Trichoptères thermophiles, voire potamiques (Caenis luctuosa, Baetis pavidus, Hydropsyche maroccana) qui caractérisent les rivières marocaines à températures estivales élevées (Dakki 1986b, Bouzidi 1989, El Agbani et al. 1992). Cependant, l'étude fait ressortir entre ces deux tronçons, un secteur de transition qui se définit non seulement par l'affrontement des assemblages précédents mais aussi par quelques espèces lénitophiles pouvant traduire, à l'image de Lymnea peregra (Chergui et al. 1999), une certaine diversité des conditions hydrauliques. De plus, un clivage faunistique très net se dégage entre tout cet ensemble amont de la Moulouya et le dernier tronçon à l'aval. La Basse-Moulouya présente en effet, associé à une très forte diversité, un cortège exclusif d'espèces potamophiles (Atyaephyra desmarestii, Psychomyia pusilla, Mystacides azurea) ou franchement limnophiles, comme les Mollusques Molanoidae (Chergui et al. 1999).

La prise en compte des groupes trophiques montre finalement que les attendus du continuum fluvial ne se vérifient que pour l'ensemble de la Moulouya à l'amont des grands barrages (Mohamed V et Mechra-Homadi). La substitution de dominance est en effet réelle : des 'shredders' de la Haute-Moulouya et des sources (secteur à la végétation abondante) aux 'grazers' dans la zone de transition incluant le haut cours des affluents d'altitudes (secteur plus large, moins ombragé), puis 
aux 'collectors' de la Moyenne-Moulouya et de ses affluents (secteur pollué à végétation très dégradée). La Basse-Moulouya, à l'aval des barrages-retenues, ne semble pas pouvoir s'intégrer dans ce continuum à moins d'invoquer ici une discontinuité touchant les groupes trophiques. Dans leur concept de discontinuités en série, Ward \& Stanford (1983) soulignent que les aménagements hydrauliques interrompent le gradient amont-aval et peuvent faire glisser les caractéristiques abiotiques et biotiques, dans un sens comme dans l'autre. Toutefois, la Basse-Moulouya se caractérise par un assemblage potamo-limnophile propre à un bas cours. De plus, si les 'grazers' dominent ici, les autres groupes trophiques ('shredders' et 'collectors') sont aussi bien représentés dans la Basse-Moulouya, alors que pourtant, la succession des groupes est déjà effective à l'amont de ce secteur. Autrement dit, la Basse-Moulouya qui est par sa faunistique typologiquement à sa place, se définit plutôt comme un tronçon indépendant du reste du cours d'eau du point de vue fonctionnel. Cet apparent paradoxe dans la BasseMoulouya est certainement lié à la combinaison des effets de-l'artificialisation (régularisation des débits avec une largeur du lit en eau réduite de moitié) et d'une situation plus naturelle quant aux ressources (plantes aquatiques et végétation riveraine ayant colonisé le lit majeur) par rapport au cours d'eau en amont. Sedell et al. (1989) ont déjà attiré l'attention sur les limites du 'R.C.C.' lorsque les interactions entre le cours d'eau et sa plaine alluviale deviennent primordiales.

En conclusion, si le 'R.C.C.' se résume aux prédictions sur la diversité faunistique et la succession des groupes fonctionnels trophiques de l'amont vers l'aval (Vannote et al. 1980, Minshall et al. 1985), cela n'est que partiellement vérifié dans le bassin de la Moulouya. La distribution longitudinale des macroinvertébrés benthiques semble dépendre ici des conditions environnementales à la fois régionales et locales. Le climat et l'hydrologie d'une part, les ressources trophiques et l'influence anthropique d'autre part (rejets domestiques dans le fleuve et impact des barrrage-retenues), apparaissent comme les facteurs structurants essentiels. Des précisions devraient être apportées sur ces facteurs, notamment en considérant les autres grands cours d'eau de la façade atlantique du Maroc (Chergui et al. 1999).

\section{Remerciements}

Nous remercions les deux lecteurs anonymes pour leurs conseils avisés ainsi que $H$. Tachet pour l'identification de certaines espèces d'Ephéméroptères, Plécoptères et Trichoptères.

\section{Références}

Berrahou A., Chavanon G., Bellouali A. \& Richoux P. 2001. Etudes sur la basse Moulouya (Maroc oriental) : 7. Les Coléoptères aquatiques de l'Oued Za. Bull. mens. Soc. linn. Lyon, 70 : sous presse.

Berrahou A., Chavanon G. \& Richoux P. 2000. - Etude sur la basse Moulouya (Maroc oriental) : 6. Les Coléoptères aquatiques de l'Oued Zegzel. Bull. mens. Soc. linn. Lyon, 69 : 44-50.

Botosaneanu L. 1979. - Quinze années de recherches sur la zonation des cours d'eau : 1963-1978 (Revue commentée de la bibliographie et observations personnelles). Bijdr. Dierk., 49 : 109-134.

Bournaud M. \& Cogérino L. 1989. — Les microhabitats aquatiques des rives d'un grand cours d'eau : approche faunistique. Ann. Limnol., 22 : 285-294.

Bouzidi A. 1989. - Recherches hydrobiologiques sur les cours d'eau des massifs du Haut Atlas (Maroc). Bio-écologie des macroinvertébrés et distribution spatiale des peuplements. Thèse, Univ. Aix-Marseille III : $185 \mathrm{p}$.

Bunn S.E. 1986. - Spatial and temporal variation in the macroinvertebrate fauna of streams in the northern jarrah forest, Western Australia : functional organisation. Freshwat. Biol., 16 : 621-632.

Cellot B., Bournaud M. \& Tachet H. 1984. - The movement of the larvae of Hydropsyche (Trichoptera) in a large river. Series Etomologica, 30 : 57-68.

Chergui H., Chavanon G., Berrahou A. \& Melahoui M. 1990. - A propos des Plécoptères du Maroc oriental. Bull. Inst. Sci. Rabat, $14: 51-53$.

Chergui H., Pattee E., Essafi K. \& Mhamdi A. 1999. - Maroccan Limnology. in Wetzel R.G. \& Gopal B. (eds). Limnology in Developing Countries, 2 : 235-330.

Cogérino L., Cellot B. \& Bournaud M. 1995. - Microhabitat diversity and associated macroinvertebrates in aquatic banks of a large European river. Hydrobiologia, 304 : 103-115.

Cummins K.W. 1973. - Trophic relations of aquatic insects. Ann. Rev. Entomol, 18 : 183-206.

Cummins K.W. 1977. - From headwater streams to rivers. Am. Biol. Teach, : 305-312.

Cummins K.W. \& Klug M.J. 1979. - Feeding ecology of stream invertebrates. Annual Review of Ecology and Systematics, 10 : 147-172.

Dakki M. 1985. - Sur le choix des données en biotypologie des eaux courantes par l'analyse factorielle des correspondances. Bull. Ecol., $16: 285-296$.

Dakki M. 1986a. - Recherches hydrobiologiques sur le haut Sebou (Moyen Atlas) ; une contribution à la connaissance faunistique, écologique et historique des eaux courantes sud-méditerranéennes. Thèse Etat, Univ. Rabat. 214 p.

Dakki M. 1986b. - Biotypologie et gradient thermique spatio-temporel, étude sur un cours d'eau du Moyen Atlas (Maroc). Bull. Ecol., $17: 79-85$.

Dia A. 1983. - Recherches sur l'écologie et la biogéographie des cours d'eau du Liban méridional. Thèse Doct., Univ. Marseille : $302 \mathrm{p}$.

Dolédec S. \& Chessel D. 1991. - Recent development in linear ordination methods for environmental sciences. Advances in Ecology, $1: 133-155$

El Agbani M.A., Dakki M. \& Bournaud M. 1992. — Etude typologique du Bou Regreg (Maroc) : Les milieux aquatiques et leurs peuplements en macroinvertébrés. Bull. Ecol., 23 : 103-113.

El Gasmi B. 1987. - Piémont du Haut Atlas de Midelt 'Maroc'. Phénomène de désertification et perspectives d'aménagement. Thèse $3^{\text {ème }}$ cycle, Univ. Paul Sabatier, Toulouse : 131 p. 
El Gharbaoui M. 1987. — Géographie physique. in : La grande encyclopédie du Maroc. Géographie physique et géologique. El Gharbaoui (ed.) Rabat : 7-13.

Gasith A. \& Resh V.H. 1999. - Streams in mediterranean climate regions : abiotic influences and biotic responses to predictable seasonal events. Ann. Rev. Ecol. Syst., 30 : 51-81.

Giudicelli J. \& Dakki M. 1984. — Les sources du Moyen Atlas et du Rif (Maroc) : faunistique (description de deux espèces nouvelles de Trichoptères), écologie, intérêt biogéographique. Bijdr. Dierk., $54: 83-100$.

Giudicelli J., Dakki M. \& Dia A. 1985. - Caractéristiques abiotiques et hydrobiologiques des eaux courantes méditerranéennes. Verh. Internat. Verein. Limnol., 22 : 2094-2101.

Giudicelli J, Dia A. \& Legier P. 1980. - Etude hydrobiologique d'une rivière de région méditerranéenne, l'Argens (Var, France) : habitats, hydrochimie, distribution de la faune benthique. Bijdr. Dierk., $50: 303-341$.

Gonzalez del Tanago M. \& Garcia de Jalon D. 1981. - Estudio de la estructura biotypologica del Rio Jamara. Bol. Est. cent. Ecol., $19: 33-51$.

Illies J. \& Botosaneanu L. 1963. - Problèmes et méthodes de la classification et de la zonation écologique des eaux courantes, considérées surtout du point de vue faunistique. Mitt. int. Verein. Limnol., 12 : 1-57.

Lounaci A., Brosse S., Thomas A. \& Lek S. 2000. - Abundance, diversity and community structure of macroinvertebrates in an Algerian stream : the Sébaou wadi. Ann. Limnol., 36 : 123-133.

Maamri A., Chergui H. \& Pattée E. 1994. - Allochthonous input of coarse particulate organic matter to a Moroccan mountain stream. Acta CEcologica, 15 : 495-508.

Marchant R., Metzeling L., Graesser A. \& Suter P. 1985. - The organization of macroinvertebrate community in the major tributaries of the La Trobe River, Victoria, Australia. Freshwat. Biol., $15: 315-331$.

Minshall G.W., Cummins K.W., Petersen R.C., Cushing C.E., Bruns D.A., Sedell J.R. \& Vannote R.L. 1985. - Development in stream ecosystem theory. Can. J. Fish. Aquat. Sci., 42 : 1045-1055.
Reynal R. 1961. - Plaines et piémonts du bassin de la Moulouya (Maroc oriental). Etude géomorphologique. Rabat : 618 p.

Rosillon D. 1986. - Evolution saisonnière de la structure fonctionnelle de la communauté benthique d'une rivière salmonicole (le Samson, Belgique). Sci. Eau, 5 : 75-84.

Sauvage C. 1963. - Les étages bioclimatiques. Notice explicative de la planche 6b de l'Atlas du Maroc,, Rabat : 31 p.

Sedell J.R., Richey J.E. \& Swanson F.J. 1989. - The river continuum concept : a basis for the expected ecosystem behavior of very large rivers? Can. Spec. Publ. Fish. Aquat. Sci., $106: 49-55$.

Statzner B. \& Higler B. 1985. - Question and comments on the river continuum concept. Can J. Fish. Aquat. Sci., 42 : 1038-1044.

Statzner B. \& Higler B. 1986. - Stream hydraulics as a major determinant of benthic invertebrate zonation patterns. Freshwat. Biol., 16 : 127-139.

Tachet H., Richoux P., Bournaud M. \& Usseglio-Polatera P. 2000. Invertébrés d'eau douce. Systématique, biologie, écologie. CNRS éditions, Paris : 588 p.

Vannote R.L., Minshall G.W., Cummins K.W., Sedell J.R. \& Cushing C.E. 1980. - The river continuum concept. Can. J. Fish. Aquat. Sci., 37 : 130-137.

Verneaux J. 1973. - Cours d'eau de Franche-Comté (massif du Jura). Recherches écologiques sur le réseau hydrographique du Doubs. Essai de biotypologie. Thèse Doct. Etat, Univ. Besançon. $260 \mathrm{p}$.

Ward J.V. \& Stanford J.A. 1983. - The serial discontinuity concept of lotic ecosystems. in Fontaine T.D.\& Bartell S.M. (eds). Dynamics of lotic ecosystems. Ann. Arbor Science, Ann Arbor, Michigan : 29-42.

Wasson J.G. 1989. - Eléments pour une typologie fonctionnelle des eaux courantes : 1 . Revue critique de quelques approches existantes. Bull. Ecol., $20: 109-127$. 
Annexe.

Appendix.

\begin{tabular}{|c|c|c|c|c|c|c|c|c|c|}
\hline & & & & & & & Mou & ouya & \\
\hline Code & Taxon & GT & s1 & $\$ 2$ & S3 & S4 & S5 & 56 & $\$ 7$ \\
\hline 1 & Dugesia gonocephala & & & & & & & & \\
\hline & Oligochaeta & $C, g$ & 1 & - & 10 & 8 & - & - & - \\
\hline 2 & Glossiphonia complanata & $\mathbf{p}$ & & - & & & & 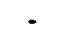 & - \\
\hline 3 & Helobdella sp. & $\mathbf{P}$ & & - & - & 9 & -. & - & - \\
\hline 4 & Erpob & $\mathbf{P}$ & - & - & 1 & - & - & - & - \\
\hline 5 & Theod & G & - & - & ; & - & - & & \\
\hline 6 & Mercuria confu & G & - & - & 1 & - & - & - & - \\
\hline 7 & Melanopsis praemorsa & G & - & - & - & - & - & - & - \\
\hline 8 & Melanopsis costellata & G & - & 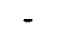 & - & - & - & 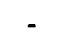 & 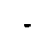 \\
\hline 9 & $A n$ & $\overline{\mathbf{G}}$ & - & - & - & - & - & - & - \\
\hline 10 & Gyra & G & - & - & - & - & - & - & - \\
\hline 11 & Phys & G & - & - & - & $\therefore$ & - & - & - \\
\hline 12 & Lymnaea & G,p & - & - & 48 & 31 & 1 & - & - \\
\hline 13 & Margaritifera margaritifera & $\mathrm{C}$ & - & - & - & & - & - & - \\
\hline 14 & Pisidium casertanum & C & - & - & - & 2 & - & 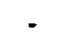 & 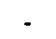 \\
\hline 15 & Gamman & $\mathbf{S}$ & 886 & 55 & - & - & - & - & - \\
\hline & (Gar & $S$ & 30 & 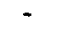 & 2 & 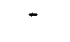 & 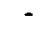 & - & \\
\hline 16 & Atyaet & $s$ & - & - & 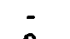 & - & - & - & - \\
\hline 17 & C & C,s & 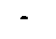 & - & 9 & 9 & - & - & : \\
\hline 18 & Caen & $\mathbf{G}$ & - & - & 9 & 4 & 3 & 3 & 1 \\
\hline 19 & Baetis neglectus & G & - & - & - & - & - & - & 12 \\
\hline 20 & Baet & G & - & 3 & 106 & 266 & 23 & 39 & 27 \\
\hline 21 & & G & 70 & 202 & - & 6 & 2 & - & 1 \\
\hline & (B: & G & - & 6 & - & - & 6 & 1 & - \\
\hline 22 & Cent & G,c & - & - & - & - & - & - & - \\
\hline 23 & Cloeon & $\mathbf{C , G}$ & . & - & 11 & - & - & - & . \\
\hline 24 & $P \boldsymbol{r}$ & $G, c$ & 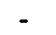 & 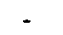 & . & - & 1 & - & 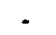 \\
\hline 25 & Oligo & $\mathrm{C}, \mathrm{g}$ & - & . & - & - & 2 & - & - \\
\hline 26 & Ecdy & (6) & & 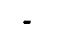 & - & - & 6 & - & 16 \\
\hline 27 & $R h i$ & G & & & - & - & 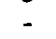 & - & 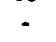 \\
\hline 28 & Rhith & G & - & - & - & - & - & - & . \\
\hline 29 & Brach & G & - & - & 5 & - & - & - & • \\
\hline 30 & Protor & $s$ & - & - & - & - & - & - & 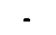 \\
\hline 31 & Perlo & S,P & - & . & 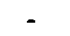 & - & - & - & 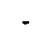 \\
\hline & Gon & $\mathbf{P}$ & - & - & - & - & 1 & . & 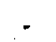 \\
\hline & Plat & P & 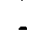 & - & . & . & 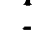 & - & - \\
\hline & & G,P & . & . & 14 & - & - & - & 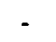 \\
\hline & & $\mathbf{P}$ & - & - & - & 3 & - & - & - \\
\hline & Meso & $\mathbf{P}$ & - & - & $=$ & - & - & - & - \\
\hline & $N$ & $\mathbf{P}$ & - & - & 1 & - & - & - & 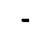 \\
\hline 32 & $A l$ & $\mathrm{P}, \mathbf{S}$ & - & 2 & 8 & 1 & 2 & 1 & 2 \\
\hline 33 & $\mathrm{Hal}$ & $\mathrm{P}, \mathrm{S}$ & - & - & - & . & - & - & 2 \\
\hline 34 & & PS & 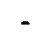 & - & $\ldots$ & . & & - & - \\
\hline 35 & & & - & & - & - & & 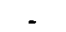 & \\
\hline 36 & & & - & 2 & 1 & 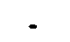 & - & 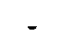 & 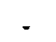 \\
\hline 37 & & $\mathrm{P}, \mathrm{S}$ & - & 4 & ii & 9 & - & - & - \\
\hline 38 & $\bar{A}_{\varepsilon}$ & P,S & - & 1 & 1 & & - & - & 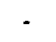 \\
\hline 39 & & $\mathbf{P}$ & - & 1 & - & - & - & - & - \\
\hline 40 & & s & - & 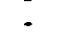 & - & - & - & . & - \\
\hline 41 & & $\vec{S}$ & - & . & 2 & 2 & 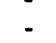 & 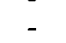 & . \\
\hline 42 & & & - & 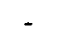 & - & 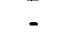 & 3 & - & - \\
\hline 43 & $E$ & $\mathbf{S}$ & - & - & - & . & . & - & - \\
\hline 44 & $m$ & $\mathbf{S}$, & 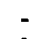 & - & - & - & - & 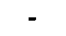 & - \\
\hline 45 & & $\mathrm{G}$ & 1 & 22 & 1 & - & - & 1 & - \\
\hline 46 & & $\mathrm{G}$ & 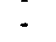 & 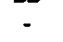 & i & . & - & 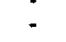 & - \\
\hline 47 & & G & - & - & - & . & - & - & - \\
\hline 48 & & G & 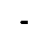 & & 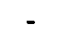 & 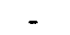 & & 2 & 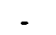 \\
\hline 49 & & G & - & - & 1 & - & 1 & - & 1 \\
\hline 50 & & G & - & - & - & - & - & - & - \\
\hline 51 & & G & - & - & 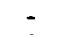 & - & - & - & - \\
\hline 52 & & G, & 2 & - & 1 & - & 2 & - & 1 \\
\hline 53 & & 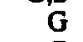 & 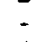 & 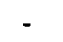 & 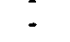 & - & - & 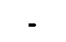 & * \\
\hline 54 & & G & 4 & - & . & - & ـ & - & - \\
\hline 55 & & G & 4 & - & - & 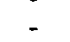 & . & 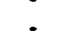 & - \\
\hline 56 & & & - & 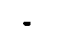 & - & 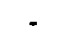 & - & 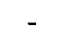 & - \\
\hline 57 & & G & . & - & - & $\because$ & . & & - \\
\hline 58 & $H$ & C & - & - & - & - & 8 & 8 & 21 \\
\hline 59 & & C & - & 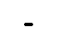 & 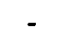 & - & 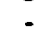 & 1 & 1 \\
\hline 60 & $H$ & C & 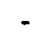 & - & - & - & - & 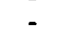 & \\
\hline 61 & & C & - & & - & - & 2 & 0 & 15 \\
\hline & & C & - & & - & . & 22 & 66 & \\
\hline 62 & & G,c & - & - & - & - & 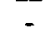 & . & 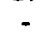 \\
\hline 63 & & $\mathrm{C}_{n} \mathrm{~s}$ & - & 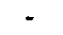 & 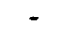 & - & - & 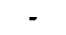 & 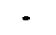 \\
\hline 64 & & P & - & - & 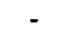 & - & 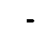 & - & - \\
\hline 65 & & $\mathbf{P}$ & & - & 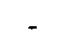 & - & - & 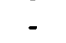 & - \\
\hline 66 & & $\mathbf{P}$ & - & - & - & - & - & - & - \\
\hline 67 & $M e$ & $S$ & 7 & 2 & 10 & 3 & & & \\
\hline 68 & & $0, \mathrm{~g}$ & 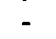 & & & & & & 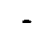 \\
\hline & & C & 74 & 214 & 44 & 978 & 88 & 1030 & 865 \\
\hline & & $\mathbf{P}$ & & & & & - & & \\
\hline & & $\mathbf{P}$ & 4 & 1 & 1 & 3 & - & 10 & 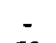 \\
\hline & ae se. & G & 17 & 15 & 292 & 240 & 3 & 872 & 53 \\
\hline & & C.s.g & 1 & 2 & 8 & 18 & 1 & 16 & . \\
\hline & & Cs & 1 & 2 & 8 & 2 & 3 & 18 & 42 \\
\hline & & $\begin{array}{l}\text { S,c,p } \\
\text {, }\end{array}$ & & & & & 2 & & 8 \\
\hline & & & - & - & . & 1 & 3 & . & 1 \\
\hline & Eph & G,S & - & & & & & & \\
\hline
\end{tabular}


Annexe. Liste et abondances totales (ind./0,3 $\mathrm{m}^{2}$ ) des macroinvertébrés récoltés début mai et fin juin 1994 dans 19 stations de la Moulouya et de ses principaux affluents (Maroc). $\mathrm{GT}=$ groupes trophiques avec $\mathrm{C}=$ 'collectors', $\mathrm{G}=$ 'grazers', $\mathrm{P}=$ 'predators', $\mathrm{S}=$ 'shredders'. En majuscules $=$ groupes principaux et en minuscules = groupes secondaires (d'après Tachet et al. 2000); exemple : Oligochaeta $C, g$ avec $C$ pour $2 / 3$ et $g$ pour $1 / 3$ des effectifs ; exemple : Tanytarsini C,s,g avec C pour $1 / 2$, s pour $1 / 4$ et $g$ pour $1 / 4$ des effectifs ; exemple : Dryops sulcipennis G,S avec $G$ pour $1 / 2$ et $S$ pour $1 / 2$ des effectifs.

Appendix. List and total abundances (ind. $/ 0.3 \mathrm{~m}^{2}$ ) of the macroinvertebrates collected in 19 sites of the Moulouya River, Morocco (from 2 periods : 2-4 May and 23-26 June 1994). $G T=$ functional feeding groups; $C=$ 'collectors' ; $G=$ 'grazers' ; $P=$ 'predators' ; $S=$ 'shredders'. Main feeding groups in capital letters, and secondary feeding groups in small letters (after Tachet et al. 2000); examples : Oligochaeta C,g with $\mathrm{C}$ $=2 / 3$ and $g=1 / 3$ of No; Tanytarsini $C, s, g$ with $C=1 / 2, s=1 / 4$ and $g=1 / 4$ of No; Dryops sulcipennis $G, S$ with $G=1 / 2$ and $S=1 / 2$ of No. 